\title{
Oxidation of sulfides using recyclable pseudocyclic benziodoxole triflate
}

\author{
Akira Yoshimura* and Viktor V. Zhdankin* \\ Department of Chemistry and Biochemistry, University of Minnesota Duluth, \\ Duluth, Minnesota, 55812, USA \\ Emails:ayoshimu@d.umn.edu,vzhdanki@d.umn.edu
}

Dedicated to Prof. Oleg A. Rakitin on the occasion of his 65th birthday

Received 09-27-2016

Accepted 10-22-2016

Published on line 12-04-2016

\section{Abstract}

A new pseudocyclic hypervalent iodine reagent, benziodoxole triflate (IBA-OTf, a complex of 2-iodosylbenzoic acid with trifluoromethanesulfonic acid), can be used as an efficient oxidant for selective oxidation of various organic sulfides to sulfoxides. This oxidation proceeds under mild condition to afford the corresponding sulfoxides in moderate to good yields without overoxidation. The reduced form of the hypervalent iodine reagent, 2-iodobenzoic acid, can be easily recovered from the reaction mixture in good yields by a simple acidbase liquid-liquid biphasic protocol.

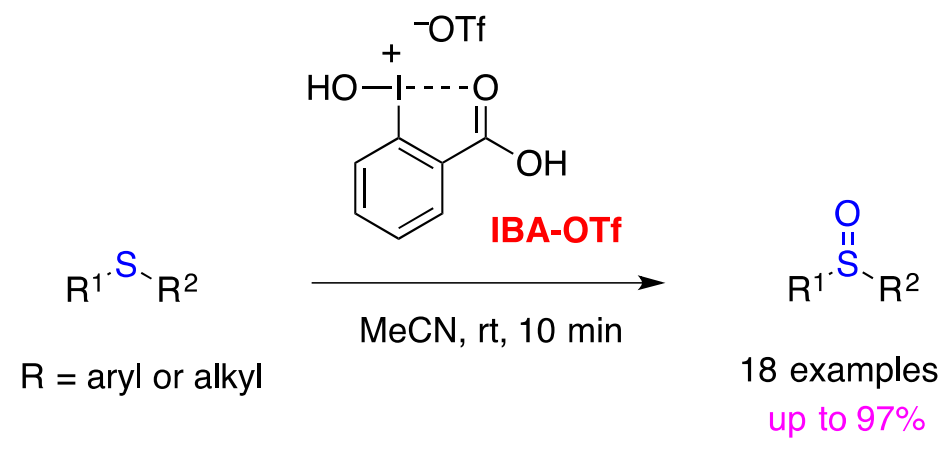

Keywords: Hypervalent iodine, benziodoxole, oxidation, sulfides, recyclable reagents 


\section{Introduction}

Hypervalent iodine compounds have found wide synthetic application as environmentally sustainable reagents for various synthetically important chemical transformations. ${ }^{1-10}$ An important class of powerful oxidants and electrophiles is represented by the triflic acid-activated hypervalent iodine reagents. In particular, [hydroxy(trifluoromethanesulfonyloxy)iodo]benzene Phl(OH)OTf can be generated in situ from iodosylbenzene and trifluoromethanesulfonic acid and used without isolation in the various oxidative transformations. ${ }^{11-18}$ However, despite its useful oxidative reactivity, Phl(OH)OTf has serious drawbacks such as low thermal stability and sensitivity to moisture. ${ }^{19}$ Recently, we have reported the synthesis and structural characterization of a new triflic acid-activated hypervalent iodine(III) reagent, benziodoxole triflate (IBA-OTf 2), which was prepared by treatment of 2-iodosylbenzoic acid (IBA 1) with trifluoromethanesulfonic acid (Scheme 1). ${ }^{20}$<smiles>O=C1OI(O)c2ccccc21</smiles>

IBA 1

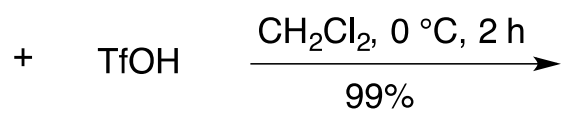

\section{(1.05 equiv)}

(a)

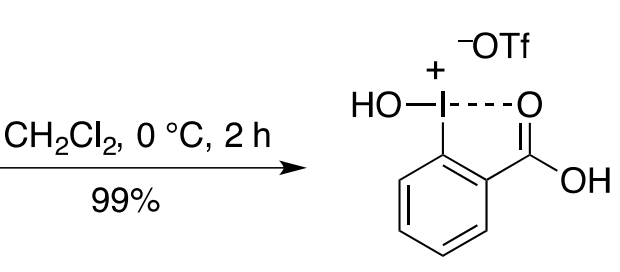

IBA-OTf 2

Scheme 1. Preparation of benziodoxole triflate 2.

IBA-OTf 2 is stable at room temperature in the presence of air and can be used as an effective electrophilic oxidizing reagent toward various organic substrates. ${ }^{21,22}$ The $X$-ray crystallographic study of IBAOTf revealed a pseudocyclic structure with strong intramolecular secondary bonding between the hypervalent iodine center and the oxygen atom of ortho-carboxylic group. The presence of this intramolecular coordination leads to the stabilization of the usually unstable hydroxy(aryl)iodonium structure. It has been documented in the literature that hypervalent iodine compounds with intramolecular secondary bonding demonstrate higher stability and improved reactivity compared to the common, noncyclic hypervalent iodine reagents. ${ }^{23-28}$ Previously, we have published preliminary results on the reactivity of IBA-OTf with various organic substrates including sulfides. ${ }^{20}$ In the present paper, we report the scope and limitations for the oxidation of organic sulfides using IBA-OTf under mild conditions. This procedure is applicable toward oxidation of alkyl- or arylsulfides bearing other sensitive functionalities and gives the corresponding sulfoxides without overoxidation.

\section{Results and Discussion}

In the initial experiment, we have found that the oxidation of thioanisole 3a using IBA-OTf $\mathbf{2}$ in acetonitrile solution at room temperature is complete in $10 \mathrm{~min}$ affording the respective sulfoxide $4 \mathrm{a}$ in $88 \%$ yield without any overoxidation to sulfone (Scheme 2). The reduced form of IBA-OTf, 2-iodobenzoic acid $\mathbf{5}$, can be easily recovered from reaction mixture in $97 \%$ by a simple acid-base liquid-liquid biphasic protocol. The recovered 2 iodobenzoic acid 5 can be easily converted to 2-iodosylbenzoic acid 1 by a standard procedure. ${ }^{29}$ 


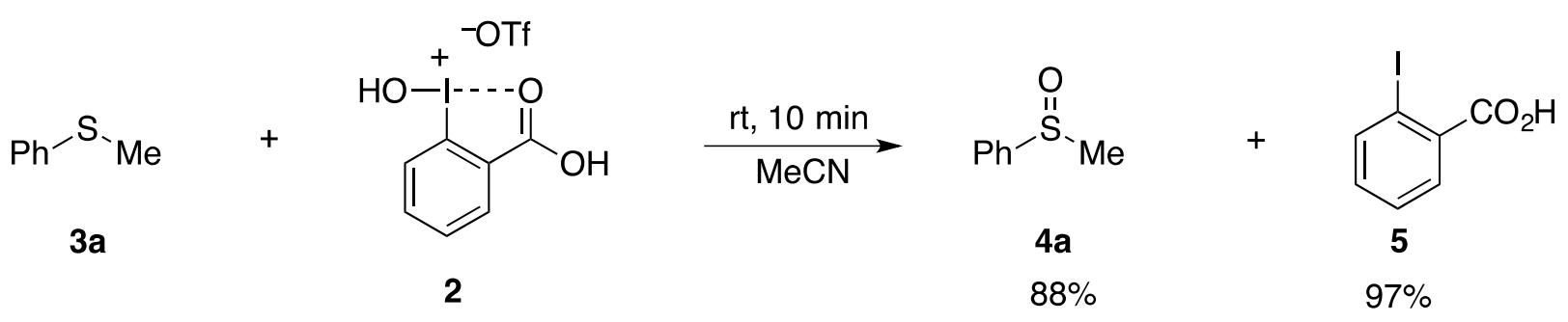

(1.2 equiv)

Scheme 2. Oxidation of thioanisole 3a using IBA-OTf 2.

Table 1. Oxidation of sulfides 3 using IBA-OTf $\mathbf{2}^{\mathrm{a}}$
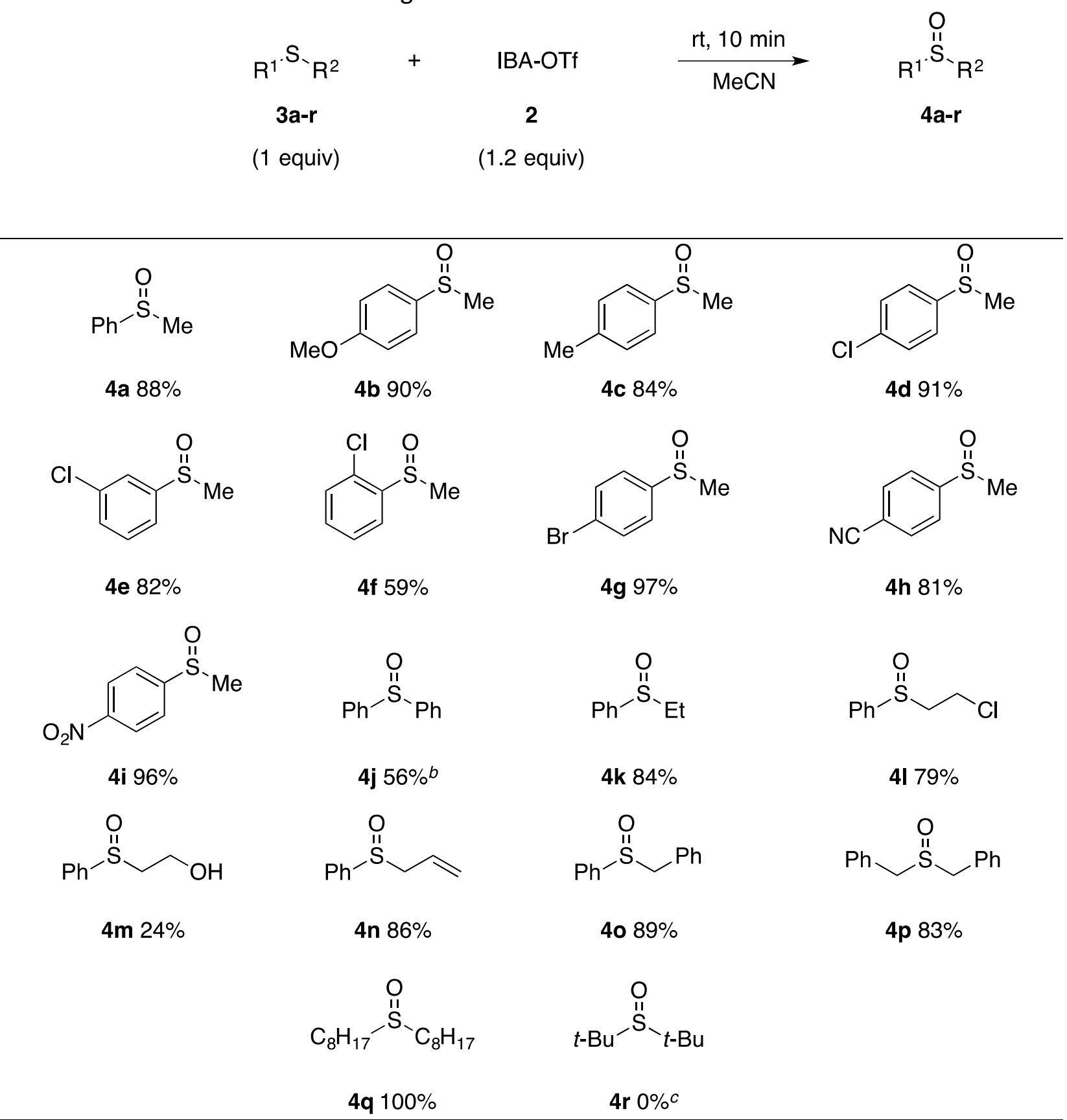

a Reaction conditions: IBA-OTf 2 (62 $\mathrm{mg}, 0.15 \mathrm{mmol})$ and sulfides 3 (0.125 mmol) in acetonitrile ( $1 \mathrm{~mL})$ at room temperature for $10 \mathrm{~min} .{ }^{\mathrm{b}}$ Reaction time was $1 \mathrm{~h}$. ${ }^{\mathrm{c}}$ Reaction time was $24 \mathrm{~h}$. 
In order to determine the scope and limitation of this reaction, we investigated the oxidation of various sulfides using IBA-OTf 2 under similar conditions (Table 1). In general, the reactions of various substituted thioanisoles 3a-i with either electron-donating or electron-withdrawing substituents in acetonitrile solution afforded the corresponding substituted sulfoxides $4 a-i$ in moderate to good yields. The reactions of phenyl alkyl sulfides $\mathbf{3 k - 0}$ under these conditions also gave the respective sulfoxides $\mathbf{4 k - 0}$ in low to good yields. As expected, the reactions of dialkyl sulfides $\mathbf{3 p - q}$ also afforded sulfoxides $\mathbf{4 p - q}$ in good yields. In the reaction of sulfides $\mathbf{3} \mathbf{f}$ and $\mathbf{3} \mathbf{j}$ with sterically hindered substituents, the corresponding sulfoxides $\mathbf{4} \mathbf{f}$ and $\mathbf{4 j}$ were obtained in relatively low yields. The most steric bulky sulfide, di-tert-butyl sulfide $\mathbf{3 r}$, did not react with IBA-OTf $\mathbf{2}$ even after $24 \mathrm{~h}$ at room temperature.

It is noteworthy that the reaction of diphenylselenide $\mathbf{6}$ using IBA-OTf $\mathbf{2}$ under same condition gave the corresponding selenoxide 7 in $65 \%$ isolated yield without overoxidation (Scheme 3). Compared to the analogous oxidation of diphenylsulfide $\mathbf{3} \mathbf{j}$, the reaction of selenide afforded the corresponding product $\mathbf{7}$ in better yield.

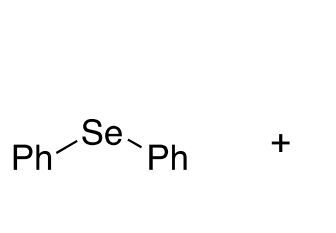

6<smiles>O=C1O[I-](O)(C(=O)O)c2ccccc21</smiles>

2<smiles>CCCC[C@@H](C)N(C)C</smiles>

7

(1.2 equiv)

Scheme 3. Oxidation of diphenylselenide 6 using IBA-OTf 2.

Finally, we have investigated a one-pot oxidation of thioanisole $\mathbf{3 a}$ using reagent $\mathbf{2}$ generated in situ from 2-iodosylbenzoic acid 1 with trifluoromethanesulfonic acid (Scheme 4). A control experiment has demonstrated that the reaction of thioanisole with IBA 1 in the absence of trifluoromethanesulfonic acid does not produce compound $\mathbf{4 a}$ and $99 \%$ of unreacted thioanisole $3 \mathbf{a}$ can be recovered from the reaction mixture (eq.1). However, when thioanisole 3a was treated with IBA 1 and trifluoromethanesulfonic acid in acetonitrile solution, product $\mathbf{4 a}$ was isolated in $50 \%$ yield (eq.2), which is a much lower yield compared to the reaction with pure reagent 2 .

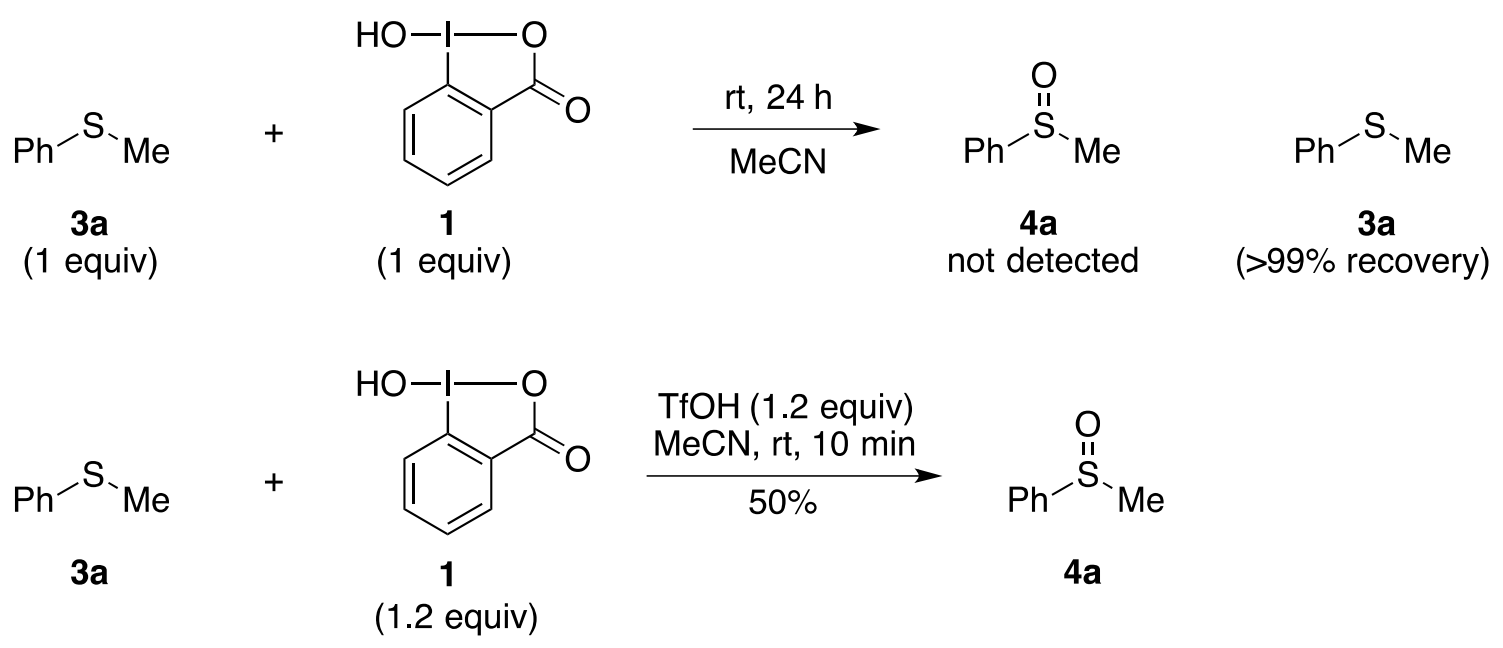

Scheme 4. One-pot oxidation of thioanisole 3a using IBA 1. 


\section{Conclusions}

We have found that IBA-OTf is an effective oxidant for the oxidation of sulfides to sulfoxides. This oxidation proceeds as a selective reaction without overoxidation to sulfones. Moreover, the reduced form of IBA-OTf, 2iodobenzoic acid, can be easily recovered from reaction mixture by a simple quenching method. This reagent can also be used for the oxidation of diphenylselenide to the corresponding selenoxide in moderate yield. Furthermore, IBA-OTf can be generated in situ from 2-iodosylbenzoic acid and trifluoromethanesulfonic acid and used for the oxidation of thioanisole.

\section{Experimental Section}

General. All reactions were performed under dry argon atmosphere with flame-dried glassware. Dichloromethane was distilled from $\mathrm{CaH}_{2}$ immediately prior to use. All commercial reagents were ACS reagent grade and used without further purification. Melting points were determined in an open capillary tube with a Mel-temp II melting point apparatus. NMR spectra were recorded on a Varian Inova $500 \mathrm{MHz}\left({ }^{1} \mathrm{H} \mathrm{NMR}\right)$. Chemical shifts are reported in parts per million (ppm) and referenced relative to the tetramethylsilane.

\section{1,3-Dihydroxy-1H-1 $\lambda^{3}$-benzo[d][1,2]iodoxol-1-yl trifluoromethanesulfonate $\quad$ (IBA-OTf)}

Trifluoromethanesulfonic acid $(0.612 \mathrm{~g}, 4.08 \mathrm{mmol})$ was added dropwise at $0{ }^{\circ} \mathrm{C}$ to a stirred mixture of 2iodosylbenzoic acid $1(1.026 \mathrm{~g}, 3.89 \mathrm{mmol})$ with $\mathrm{CH}_{2} \mathrm{Cl}_{2}(5 \mathrm{~mL})$. The reaction was stirred at $0{ }^{\circ} \mathrm{C}$ for $2 \mathrm{~h}$. After completion of the reaction, the solvent was removed under reduced pressure and the solid product was washed with hexane and diethyl ether several times then dried in vacuum to give $1.593 \mathrm{~g}$ (99\%) of compound 2 as a white solid. Recrystallization of product 4 from acetonitrile at $0{ }^{\circ} \mathrm{C}$ afforded colorless prisms; $\mathrm{mp} 154.6$ $155.1^{\circ} \mathrm{C} ;{ }^{1} \mathrm{H}$ NMR $\left(500 \mathrm{MHz}, \mathrm{CD}_{3} \mathrm{CN}\right): \delta 8.38(\mathrm{~d}, J 7.8 \mathrm{~Hz} 1 \mathrm{H}), 8.25-8.19(\mathrm{~m}, 1 \mathrm{H}), 8.02(\mathrm{~d}, J 8.5 \mathrm{~Hz}, 1 \mathrm{H}), 7.94-7.89$ $(\mathrm{m}, 1 \mathrm{H})$.

General procedure for oxidation of sulfides to sulfoxides using IBA-OTf 2. Sulfide 3 (0.125 mmol) was added to a solution of $2(62 \mathrm{mg}, 0.15 \mathrm{mmol})$ in acetonitrile $(1 \mathrm{~mL})$. The reaction was stirred at room temperature for $10 \mathrm{~min}$ to $1 \mathrm{~h}$ (reaction completion was controlled by TLC). After completion of the reaction, 5\% aqueous $\mathrm{Na}_{2} \mathrm{~S}_{2} \mathrm{O}_{3}(5 \mathrm{~mL})$ and saturated $\mathrm{NaHCO}_{3}(5 \mathrm{~mL})$ were added, and the mixture was extracted with dichloromethane. The organic phase was dried over anhydrous $\mathrm{Na}_{2} \mathrm{SO}_{4}$ and concentrated under reduced pressure. Purification using short chromatographic column (hexane-ethyl acetate $=3: 1$ ) afforded analytically pure sulfoxide 4.

Recovery of 2-iodobenzoic acid 5: The aqueous layer was acidified with $12 \mathrm{M} \mathrm{HCl}$ to $\mathrm{pH}$ about 1-2 and then extracted with dichloromethane. The organic phase was dried over anhydrous $\mathrm{Na}_{2} \mathrm{SO}_{4}$ and concentrated under reduced pressure to give $36 \mathrm{mg}(97 \%)$ of 2 -iodobenzoic acid 5.

Methyl phenyl sulfoxide (4a). ${ }^{20}$ Reaction of thioanisole $3 a(16 \mathrm{mg}, 0.125 \mathrm{mmol}$ ) according to general procedure afforded $15 \mathrm{mg}(83 \%)$ of product $4 \mathrm{a}$ as a yellow oil: ${ }^{1} \mathrm{H} \mathrm{NMR}\left(500 \mathrm{MHz}, \mathrm{CDCl}_{3}\right): \delta 7.68-7.63(\mathrm{~m}, 2 \mathrm{H})$, 7.57-7.48 (m, 3H), $2.73(\mathrm{~s}, 3 \mathrm{H})$.

p-Methoxy phenyl methyl sulfoxide (4b). ${ }^{30}$ Reaction of $p$-methoxyphenyl methyl sulfide $\mathbf{3 b}$ (19 mg, 0.125 mmol) according to general procedure afforded $19 \mathrm{mg}(90 \%)$ of product $\mathbf{4 b}$ as a light yellow oil: ${ }^{1} \mathrm{H}$ NMR (500 $\left.\mathrm{MHz}, \mathrm{CDCl}_{3}\right): \delta 7.58(\mathrm{~d}, J 8.8, \mathrm{~Hz}, 2 \mathrm{H}), 7.02(\mathrm{~d}, J 8.8 \mathrm{~Hz}, 2 \mathrm{H}), 3.84(\mathrm{~s}, 3 \mathrm{H}), 2.68(\mathrm{~s}, 3 \mathrm{H})$. 
Methyl $p$-tolyl sulfoxide (4c). ${ }^{31}$ Reaction of methyl $p$-tolyl sulfide $3 c(17 \mathrm{mg}, 0.125 \mathrm{mmol}$ ) according to general procedure afforded $16 \mathrm{mg}(84 \%)$ of product $4 \mathrm{c}$ as a yellow oil: ${ }^{1} \mathrm{H} \mathrm{NMR}\left(500 \mathrm{MHz}, \mathrm{CDCl}_{3}\right): \delta 7.52(\mathrm{~d}, J 7.5 \mathrm{~Hz}$, $1 \mathrm{H}), 7.31$ (d, J $7.5 \mathrm{~Hz}, 1 \mathrm{H}), 2.69(\mathrm{~s}, 3 \mathrm{H}), 2.40(\mathrm{~s}, 3 \mathrm{H})$.

p-Chlorophenyl methyl sulfoxide (4d). ${ }^{30}$ Reaction of $p$-chlorophenyl methyl sulfide $\mathbf{3 d}(20 \mathrm{mg}, 0.125 \mathrm{mmol})$ according to general procedure afforded $20 \mathrm{mg}(91 \%)$ of product $4 \mathbf{d}$ as a yellow oil: ${ }^{1} \mathrm{H} \mathrm{NMR}\left(500 \mathrm{MHz}, \mathrm{CDCl}_{3}\right)$ : $\delta 7.60(\mathrm{~d}, J 8.0 \mathrm{~Hz}, 2 \mathrm{H}), 7.52(\mathrm{~d}, J 8.0 \mathrm{~Hz}, 2 \mathrm{H}), 2.72(\mathrm{~s}, 3 \mathrm{H})$.

$\boldsymbol{m}$-Chlorophenyl methyl sulfoxide (4e). ${ }^{32}$ Reaction of $m$-chlorophenyl methyl sulfide $3 e(20 \mathrm{mg}, 0.125 \mathrm{mmol})$ according to general procedure afforded $18 \mathrm{mg}(82 \%)$ of product $4 \mathrm{e}$ as a colorless oil: ${ }^{1} \mathrm{H} \mathrm{NMR}(500 \mathrm{MHz}$, $\left.\mathrm{CDCl}_{3}\right): \delta 7.66(\mathrm{~s}, 1 \mathrm{H}), 7.52-7.44(\mathrm{~m}, 3 \mathrm{H}), 2.74(\mathrm{~s}, 3 \mathrm{H})$.

o-Chlorophenyl methyl sulfoxide (4f). ${ }^{33}$ Reaction of $o$-chlorophenyl methyl sulfide $\mathbf{3 f}(20 \mathrm{mg}, 0.125 \mathrm{mmol})$ according to general procedure afforded $13 \mathrm{mg}(59 \%)$ of product $\mathbf{4 f}$ as a colorless oil: ${ }^{1} \mathrm{H} \mathrm{NMR}(500 \mathrm{MHz}$, $\left.\mathrm{CDCl}_{3}\right): \delta 7.97(\mathrm{dd}, J 8.0 \mathrm{~Hz}, 1.5 \mathrm{~Hz}, 1 \mathrm{H}), 7.57-7.52(\mathrm{~m}, 1 \mathrm{H}), 7.45(\mathrm{dt}, J 7.8 \mathrm{~Hz}, 1.5 \mathrm{~Hz}, 1 \mathrm{H}), 7.40(\mathrm{~d}, J 7.5 \mathrm{~Hz}, 1 \mathrm{H})$, $2.83(\mathrm{~s}, 3 \mathrm{H})$.

p-Bromophenyl methyl sulfoxide (4g) ${ }^{30}$ Reaction of $p$-bromophenyl methyl sulfide $3 \mathrm{~g}(25 \mathrm{mg}, 0.125 \mathrm{mmol})$ according to general procedure afforded $26 \mathrm{mg}(97 \%)$ of product $4 \mathrm{~g}$ as a white solid: $\mathrm{mp} 84.3-84.6{ }^{\circ} \mathrm{C}$ (lit. ${ }^{30}$; mp 82.4-83.3 $\left.{ }^{\circ} \mathrm{C}\right):{ }^{1} \mathrm{H}$ NMR $\left(500 \mathrm{MHz}, \mathrm{CDCl}_{3}\right): \delta 7.66(\mathrm{~d}, J 8.3 \mathrm{~Hz}, 2 \mathrm{H}), 7.51(\mathrm{~d}, J 8.3 \mathrm{~Hz}, 2 \mathrm{H}), 2.71(\mathrm{~s}, 3 \mathrm{H})$.

p-Cyanophenyl methyl sulfoxide (4h). ${ }^{34}$ Reaction of $p$-cyanophenyl methyl sulfide $3 \mathrm{~h}$ (19 $\left.\mathrm{mg}, 0.125 \mathrm{mmol}\right)$ according to general procedure afforded $17 \mathrm{mg}(81 \%)$ of product $4 \mathrm{~h}$ as a white solid: $\mathrm{mp} 91.2-91.5^{\circ} \mathrm{C}$ (lit. ${ }^{34}$; $\left.\mathrm{mp} 87-88^{\circ} \mathrm{C}\right):{ }^{1} \mathrm{H}$ NMR $\left(500 \mathrm{MHz}, \mathrm{CDCl}_{3}\right): \delta 7.82$ (d, J $\left.8.3 \mathrm{~Hz}, 2 \mathrm{H}\right), 7.76(\mathrm{~d}, J 8.3 \mathrm{~Hz}, 2 \mathrm{H}), 2.76(\mathrm{~s}, 3 \mathrm{H})$.

p-Nitrophenyl methyl sulfoxide (4i). ${ }^{35}$ Reaction of $p$-nitrophenyl methyl sulfide $3 \mathbf{i}(21 \mathrm{mg}, 0.125 \mathrm{mmol})$ according to general procedure afforded $22 \mathrm{mg}(96 \%)$ of product $4 \mathbf{i}$ as a white solid: $\mathrm{mp} 150.5-150.8^{\circ} \mathrm{C}$ (lit. ${ }^{35}$; mp 153.0-155.0 $\left.{ }^{\circ} \mathrm{C}\right):{ }^{1} \mathrm{H} N M R\left(500 \mathrm{MHz}, \mathrm{CDCl}_{3}\right): \delta 8.38(\mathrm{~d}, J 9.0 \mathrm{~Hz}, 2 \mathrm{H}), 7.83(\mathrm{~d}, J 9.0 \mathrm{~Hz}, 2 \mathrm{H}), 3.79(\mathrm{~s}, 3 \mathrm{H})$.

Diphenyl sulfoxide (4j). ${ }^{35}$ Reaction of diphenyl sulfide $3 \mathbf{i}(23 \mathrm{mg}, 0.125 \mathrm{mmol})$ according to general procedure afforded $14 \mathrm{mg}$ (56\%) of product $4 \mathbf{i}$ as a colorless oil: ${ }^{1} \mathrm{H} N M R\left(500 \mathrm{MHz}, \mathrm{CDCl}_{3}\right): \delta 7.78-7.63(\mathrm{~m}, 4 \mathrm{H}), 7.50-7.41$ $(\mathrm{m}, 6 \mathrm{H})$.

Phenyl ethyl sulfoxide (4k). ${ }^{30}$ Reaction of phenyl ethyl sulfide $\mathbf{3 k}(17 \mathrm{mg}, 0.125 \mathrm{mmol}$ ) according to general procedure afforded $16 \mathrm{mg}(84 \%)$ of product $\mathbf{4 k}$ as a colorless oil: ${ }^{1} \mathrm{H} \mathrm{NMR}\left(500 \mathrm{MHz}, \mathrm{CDCl}_{3}\right): \delta 7.63-7.59(\mathrm{~m}$, $2 \mathrm{H})$, 7.55-7.47 (m,3H), 2.95-2.86 (m, 1H), 2.81-2.72 (m, 1H), $1.20(\mathrm{t}, J 7.5 \mathrm{~Hz}, 3 \mathrm{H})$.

2-Chloroethyl phenyl sulfoxide (4l). ${ }^{30}$ Reaction of 2-chloroethyl phenyl sulfide 31 (22 $\mathrm{mg}, 0.125 \mathrm{mmol}$ ) according to general procedure afforded $19 \mathrm{mg}(79 \%)$ of product $4 \mathrm{l}$ as a colorless oil: ${ }^{1} \mathrm{H} \mathrm{NMR}(500 \mathrm{MHz}$, $\left.\mathrm{CDCl}_{3}\right): \delta 7.64(\mathrm{~d}, J 7.5 \mathrm{~Hz}, 2 \mathrm{H}), 7.58-7.50(\mathrm{~m}, 3 \mathrm{H}), 4.00-3.93(\mathrm{~m}, 1 \mathrm{H}), 3.69-3.62(\mathrm{~m}, 1 \mathrm{H}), 3.22-3.11(\mathrm{~m}, 2 \mathrm{H})$.

2-Phenyl sulfoxy ethanol $(\mathbf{4 m}){ }^{35}$ Reaction of 2-(phenylthio)ethanol $3 \mathrm{~m} \mathrm{(19} \mathrm{mg}, 0.125 \mathrm{mmol}$ ) according to general procedure afforded $5 \mathrm{mg}(24 \%)$ of product $4 \mathrm{~m}$ as a colorless oil: $\left.{ }^{1} \mathrm{H} \mathrm{NMR} \mathrm{(500} \mathrm{MHz,} \mathrm{CDCl}_{3}\right): \delta 7.66(\mathrm{dd}$, J $8.3 \mathrm{~Hz}, 1.3 \mathrm{~Hz}, 2 \mathrm{H}), 7.60-7.51(\mathrm{~m}, 3 \mathrm{H}), 4.21-4.12(\mathrm{~m}, 1 \mathrm{H}), 4.08-4.02(\mathrm{~m}, 1 \mathrm{H}), 3.26-3.19(\mathrm{~m}, 1 \mathrm{H}), 2.90-2.83(\mathrm{~m}$, $1 \mathrm{H})$.

Allyl phenyl sulfoxide (4n). ${ }^{35}$ Reaction of allyl phenyl sulfide $3 \mathrm{n}$ (19 $\mathrm{mg}, 0.125 \mathrm{mmol}$ ) according to general procedure afforded $18 \mathrm{mg}(86 \%)$ of product $4 \mathrm{n}$ as a colorless oil: ${ }^{1} \mathrm{H} \mathrm{NMR}\left(500 \mathrm{MHz}, \mathrm{CDCl}_{3}\right): \delta 7.62-7.57(\mathrm{~m}$, $2 \mathrm{H})$, 7.54-7.46 (m, 3H), 5.70-5.58 (m, $1 \mathrm{H}), 5.33(\mathrm{~d}, J 11.0 \mathrm{~Hz}, 1 \mathrm{H}), 5.19(\mathrm{~d}, J 17.0 \mathrm{~Hz}, 1 \mathrm{H}), 3.61-3.46(\mathrm{~m}, 2 \mathrm{H})$.

Benzyl phenyl sulfoxide (40). ${ }^{30,36}$ Reaction of benzyl phenyl sulfide 30 (25 mg, $0.125 \mathrm{mmol}$ ) according to general procedure afforded $24 \mathrm{mg}(89 \%)$ of product 40 as a white solid: $\mathrm{mp} 123.2-123.4^{\circ} \mathrm{C}$ (lit. ${ }^{30}$; mp 122.4 $\left.123.6{ }^{\circ} \mathrm{C}\right):{ }^{1} \mathrm{H}$ NMR $\left(500 \mathrm{MHz}, \mathrm{CDCl}_{3}\right): \delta 7.49-7.36(\mathrm{~m}, 5 \mathrm{H}), 7.32-7.22(\mathrm{~m}, 3 \mathrm{H}), 6.99(\mathrm{~d}, J 7.0 \mathrm{~Hz}, 2 \mathrm{H}), 4.10(\mathrm{~d}, J 7.8$ $\mathrm{Hz}, 1 \mathrm{H}), 4.00(\mathrm{~d}, J 7.8 \mathrm{~Hz}, 1 \mathrm{H})$. 
Dibenzylsulfoxide (4p). ${ }^{37}$ Reaction of dibenzylsulfide $3 p(27 \mathrm{mg}, 0.125 \mathrm{mmol}$ ) according to general procedure afforded $24 \mathrm{mg}$ (83\%) of product $4 \mathrm{p}$ as a white solid: $\mathrm{mp} 128.1-128.3^{\circ} \mathrm{C}$ (lit. ${ }^{36} ; \mathrm{mp} 129.0-131.0{ }^{\circ} \mathrm{C}$ ): ${ }^{1} \mathrm{H} \mathrm{NMR}$ (500 MHz, $\left.\mathrm{CDCl}_{3}\right): \delta 7.41-7.27(\mathrm{~m}, 10 \mathrm{H}), 3.93(\mathrm{~d}, J 8.0 \mathrm{~Hz}, 2 \mathrm{H}), 3.87(\mathrm{~d}, J 8.0 \mathrm{~Hz}, 2 \mathrm{H})$.

Dioctylsulfoxide (4q). ${ }^{30}$ Reaction of dioctylsulfide $\mathbf{3 q}(32 \mathrm{mg}, 0.125 \mathrm{mmol}$ ) according to general procedure afforded $34 \mathrm{mg}(100 \%)$ of product $4 \mathrm{q}$ as a white solid: $\mathrm{mp} 72.2-72.7^{\circ} \mathrm{C}$ (lit. $\left.{ }^{30}, \mathrm{mp} 75.0-76.0{ }^{\circ} \mathrm{C}\right):{ }^{1} \mathrm{H} \mathrm{NMR}(500$ $\left.\mathrm{MHz}, \mathrm{CDCl}_{3}\right): \delta 2.72-2.57(\mathrm{~m}, 4 \mathrm{H}), 1.82-1.70(\mathrm{~m}, 4 \mathrm{H}), 1.53-1.20(\mathrm{~m}, 2 \mathrm{H}), 0.88(\mathrm{t}, J 7.0 \mathrm{~Hz}, 6 \mathrm{H})$.

Oxidation of diphenylselenide 6 to diphenylselenoxide 7 using IBA-OTf 2. Diphenylselenide 6 (29 mg, 0.125 $\mathrm{mmol}$ ) was added to a solution of $2(62 \mathrm{mg}, 0.15 \mathrm{mmol})$ in acetonitrile $(1 \mathrm{~mL})$. The reaction was stirred at room temperature for $1 \mathrm{~h}$ (reaction completion was controlled by TLC). After completion of the reaction, $5 \%$ aqueous $\mathrm{Na}_{2} \mathrm{~S}_{2} \mathrm{O}_{3}(5 \mathrm{~mL})$ and saturated $\mathrm{NaHCO}_{3}(5 \mathrm{~mL})$ were added, and the mixture was extracted with dichloromethane. The organic phase was dried over anhydrous $\mathrm{Na}_{2} \mathrm{SO}_{4}$ and concentrated under reduced pressure. Purification by short column chromatography (hexane-ethyl acetate $3: 1$ ) afforded analytically pure diphenylselenoxide 7; $20 \mathrm{mg}$ (65\%) isolated as a brown solid: $\mathrm{mp} 110.0-111.6{ }^{\circ} \mathrm{C}\left(\right.$ lit. $\left.{ }^{38}, \mathrm{mp} 100.0-101.0{ }^{\circ} \mathrm{C}\right):{ }^{1} \mathrm{H}$ NMR $\left(500 \mathrm{MHz} \mathrm{CDCl}_{3}\right): \delta$ 7.72-7.67 $(\mathrm{m}, 4 \mathrm{H}), 7.49-7.42(\mathrm{~m}, 6 \mathrm{H})$.

One-pot oxidation of thioanisole 3a using IBA-OTf 1 and trifluoromethanesulfonic acid. Thioanisole 3a (16 $\mathrm{mg}, 0.125 \mathrm{mmol}$ ), and trifluoromethanesulfonic acid (23 mg, $0.150 \mathrm{mmol}$ ) were added to a solution of 1 (40 $\mathrm{mg}, 0.150 \mathrm{mmol}$ ) in acetonitrile $(1 \mathrm{~mL}$ ). The reaction was stirred at room temperature for $1 \mathrm{~h}$ (reaction completion was controlled by TLC). After completion of the reaction, $5 \%$ aqueous $\mathrm{Na}_{2} \mathrm{~S}_{2} \mathrm{O}_{3}(5 \mathrm{~mL})$ and saturated $\mathrm{NaHCO}_{3}(5 \mathrm{~mL})$ were added, and the mixture was extracted with dichloromethane. The organic phase was dried over anhydrous $\mathrm{Na}_{2} \mathrm{SO}_{4}$ and concentrated under reduced pressure. Purification by short column chromatography (hexane-ethyl acetate = $3: 1$ ) afforded analytically pure product $4 \mathrm{a} ; 9 \mathrm{mg}$ (50\%) isolated as a yellow oil identical to the sample from previous experiment.

\section{Acknowledgements}

This work was supported by a research grant from the NSF (CHE-1262479).

\section{References}

$1 \quad$ Wirth, T.; Ed. Hypervalent lodine Chemistry [In: Top. Curr. Chem., 2016, 373], 2016.

2 Zhdankin, V. V. Hypervalent lodine Chemistry: Preparation, Structure, and Synthetic Applications of Polyvalent lodine Compounds; Wiley: Chichester (UK), 2013.

http://dx.doi.org/10.1002/9781118341155

3 Yoshimura, A.; Zhdankin, V. V. Chem. Rev. 2016, 116, 3328-3435.

http://dx.doi.org/10.1021/acs.chemrev.5b00547

4 Dohi, T.; Kita, Y. Curr. Org. Chem. 2016, 20, 580-615.

http://dx.doi.org/10.2174/1385272819666150716173142

5 Yusubov, M. S.; Yoshimura, A.; Zhdankin, V. V. Arkivoc 2016, (i), 342-374.

http://dx.doi.org/10.3998/ark.5550190.p009.732

6 Li, Y.; Hari, D. P.; Vita, M. V.; Waser, J. Angew. Chem., Int. Ed. 2016, 55, 4436-4454. http://dx.doi.org/10.1002/anie.201509073

7 Singh, F. V.; Wirth, T. Chem. - Asian J. 2014, 9, 950-971. 
http://dx.doi.org/10.1002/asia.201301582

8 Romero, R. M.; Woeste, T. H.; Muniz, K. Chem. - Asian J. 2014, 9, 972-983.

http://dx.doi.org/10.1002/asia.201301637

9 Yusubov, M. S.; Zhdankin, V. V. Mendeleev Commun. 2010, 20, 185-191.

http://dx.doi.org/10.1016/i.mencom.2010.06.001

10 Merritt, E. A.; Olofsson, B. Angew. Chem., Int. Ed. 2009, 48, 9052-9070.

http://dx.doi.org/10.1002/anie.200904689

11 Kitamura, T.; Furuki, R.; Taniguchi, H.; Stang, P. J. Tetrahedron Lett. 1990, 31, 703-704.

http://dx.doi.org/10.1016/S0040-4039(00)94607-0

12 Kitamura, T.; Furuki, R.; Taniguchi, H.; Stang, P. J. Tetrahedron 1992, 48, 7149-7156.

http://dx.doi.org/10.1016/S0040-4020(01)88255-7

13 Saito, A.; Taniguchi, A.; Kambara, Y.; Hanzawa, Y. Org. Lett. 2013, 15, 2672-2675.

http://dx.doi.org/10.1021/ol4009816

14 Kitamura, T.; Zheng, L.; Fukuoka, T.; Fujiwara, Y.; Taniguchi, H.; Sakurai, M.; Tanaka, R. J. Chem. Soc.,

Perkin Trans. 2 1997, 1511-1515.

http://dx.doi.org/10.1039/a700602k

15 Kitamura, T. Yuki Gosei Kagaku Kyokaishi 1995, 53, 893-905.

http://dx.doi.org/10.5059/yukigoseikyokaishi.53.893

16 Saito, A.; Hyodo, N.; Hanzawa, Y. Molecules 2012, 17, 11046-11055.

http://dx.doi.org/10.3390/molecules170911046

17 Kitamura, T.; Matsuyuki, J.; Nagata, K.; Furuki, R.; Taniguchi, H. Synthesis 1992, 945-946.

http://dx.doi.org/10.1055/s-1992-26272

18 Meier, R.; Strych, S.; Trauner, D. Org. Lett. 2014, 16, 2634-2637.

http://dx.doi.org/10.1021/ol500800z

19 Kitamura, T.; Nagata, K.; Nakamura, T.; Furuki, R.; Taniguchi, H. Tetrahedron 1995, 51, 6229-6236.

http://dx.doi.org/10.1016/0040-4020(95)00280-L

20 Yoshimura, A.; Nguyen, K. C.; Klasen, S. C.; Saito, A.; Nemykin, V. N.; Zhdankin, V. V. Chem. Commun. 2015, 51, 7835-7838.

http://dx.doi.org/10.1039/C5CC02009C

21 Yoshimura, A.; Nguyen, K. C.; Rohde, G. T.; Saito, A.; Yusubov, M. S.; Zhdankin, V. V. Adv. Synth. Catal. 2016, 358, 2340-2344.

http://dx.doi.org/10.1002/adsc.201600331

22 Yoshimura, A.; Nguyen, K. C.; Klasen, S. C.; Postnikov, P. S.; Yusubov, M. S.; Saito, A.; Nemykin, V. N.;

Zhdankin, V. V. Asian J. Org. Chem. 2016, 5, 1128-1133.

http://dx.doi.org/10.1002/ajoc.201600247

23 Yoshimura, A.; Yusubov, M. S.; Zhdankin, V. V. Org. Biomol. Chem. 2016, 14, 4771-4781. http://dx.doi.org/10.1039/C6OB00773B

24 Zhdankin, V. V.; Protasiewicz, J. D. Coord. Chem. Rev. 2014, 275, 54-62.

http://dx.doi.org/10.1016/j.ccr.2014.04.007

25 Meprathu, B. V.; Protasiewicz, J. D. Tetrahedron 2010, 66, 5768-5774.

http://dx.doi.org/10.1016/j.tet.2010.04.087

26 Yoshimura, A.; Nemykin, V. N.; Zhdankin, V. V. Chem.--Eur. J. 2011, 17, 10538-10541.

http://dx.doi.org/10.1002/chem.201102265

27 Zhu, C.; Yoshimura, A.; Ji, L.; Wei, Y.; Nemykin, V. N.; Zhdankin, V. V. Org. Lett. 2012, 14, 3170-3173. 
http://dx.doi.org/10.1021/ol301268j

28 Hamnett, D. J.; Moran, W. J. Org. Biomol. Chem. 2014, 12, 4156-4162.

http://dx.doi.org/10.1039/c4ob00556b

29 Fernandez Gonzalez, D.; Brand, J. P.; Waser, J. Chem.--Eur. J. 2010, 16, 9457-9461. http://dx.doi.org/10.1002/chem.201001539

30 Yoshimura, A.; Banek, C. T.; Yusubov, M. S.; Nemykin, V. N.; Zhdankin, V. V. J. Org. Chem. 2011, 76, 3812-3819.

http://dx.doi.org/10.1021/jo070700n

31 Shaabani, A.; Lee, D. G. Synth. Commun. 2003, 33, 1845-1854.

http://dx.doi.org/10.1081/SCC-120020194

32 Hanson, P.; Hendrickx, R. A. A. J.; Smith, J. R. L. Org. Biomol. Chem. 2008, 6, 745-761. http://dx.doi.org/10.1039/b714707d

33 Zhang, H.; Chen, C.; Liu, R. Synth. Commun. 2012, 42, 811-819.

http://dx.doi.org/10.1080/00397911.2010.531439

34 Imada, Y.; Takagishi, M.; Komiya, N.; Naota, T. Synth. Commun. 2013, 43, 3064-3071.

http://dx.doi.org/10.1080/00397911.2013.767912

35 Zhao, L.; Zhang, H.; Wang, Y. J. Org. Chem. 2016, 81, 129-136.

http://dx.doi.org/10.1021/acs.joc.5b02400

36 Koposov, A. Y.; Zhdankin, V. V. Synthesis 2005, 22-24.

http://dx.doi.org/10.1055/s-2004-834873

37 Boudou, C.; Berges, M.; Sagnes, C.; Sopkova-de Oliveira Santos, J.; Perrio, S.; Metzner, P. J. Org. Chem. 2007, 72, 5403-5406

http://dx.doi.org/10.1021/jo070700n

38 Oba, M.; Okada, Y.; Endo, M.; Tanaka, K.; Nishiyama, K.; Shimada, S.; Ando, W. Inorg. Chem. 2010, 49, 10680-10686.

http://dx.doi.org/10.1021/ic101708y 